\title{
Molecular spectroscopy analysis of the substitution of bone tissue by HAp/PLLA composite biomaterial
}

\author{
Nenad Ignjatovic and Dragan Uskokovic* \\ Institute of Technical Sciences of the Serbian Academy of Sciences and Arts, K. Mihailova 35/IV, \\ 11000 Belgrade, Yugoslavia
}

\begin{abstract}
Due to its pronounced osteoinductive properties, calcium hydroxyapatite (HAp) has been widely used in medicine. Bioresorptive poly-L-lactide (PLLA) as a polymer biomaterial has been also used extensively in medicine for its non-toxicity and biocompatibility. To combine the advantages exhibited by each of these materials, a HAp/PLLA composite biomaterial has been synthesized and used for reconstruction and repair of bone defects. Hydroxyapatite/poly-L-lactide (HAp/PLLA) composite biomaterial with PLLA of 50,000 and 430,000 g/mole molecular weight was studied in vivo. The biocomposite with PLLA of both molecular weights was implanted into mice, then removed from their organisms and analyzed by the Fourier transform infrared (FT-IR) spectroscopy, scanning electron microscopy (SEM) and histopathologic analysis. Characteristic absorption bands, registered and defined by FT-IR spectroscopy, confirm the formation of new functional groups and compounds during the bone repair process using HAp/PLLA biocomposite with PLLA of 50,000 and 430,000 molecular weights. Analysis of the microstructures of the sample surfaces by scanning electron microscopy (SEM) before and after implantation revealed bioresorption of the PLLA polymer phase in the system with PLLA of lower molecular weight and generation of collagen fibers at the sites of implanted bioresorptive PLLA. As the studied synthetic materials behave as the natural bone, i.e., they are phagocytosed and resorpable, they can be considered as biocompatible.
\end{abstract}

\section{Introduction}

The reconstruction of bone tissue may be done by various biomaterials, which must satisfy some basic requirements such as to be nontoxic, biocompatible, biodegradable and bioresorpable. In the case where the bone tissue interruptions are present, the implant materials must have good mechanical properties. The synthesized calcium hydroxyapatite (HAp), being a very similar to the inorganic phase in bone tissue, has been used as osteoconductive ceramic material [1]. Poly-L-lactide (PLLA) has been also widely employed to date in medicine and stomatology, because of its biocompatible, nontoxic and bioresorpable properties [2,3]. It has been reported [4,5] that the inorganic apatite particles or powder can be effectively reinforced by polymer PLLA and that the obtained biocomposite HAp/PLLA exhibits favorable properties of both components. The inorganic component (HAp) provides a nonresorbable and osteoconductive properties and polymer component (PLLA) provides a bioresorpable properties to the

\footnotetext{
${ }^{*}$ Corresponding author: Prof. Dr D. Uskokovic, Institute of Technical Sciences of the Serbian Academy of Sciences and Arts, K. Mihailova 35/IV, 11000 Belgrade, Yugoslavia. Tel.: +381 11 636994, +381 11 185 437; Fax: +381 11 185-263; E-mail: uskok @itn.sanu.ac.yu.
} 
biocomposite system. During the implantation process, PLLA in contact with the natural environment tissue, being bioresorpable, creates a sites for new-formed tissue by organism.

Various methods can be employed to obtain HAp/PLLA biocomposites. By addition of HAp into the L-lactide monomer, and subsequent monomer polymerization, HAp/PLLA composite biomaterial can be obtained [6]. Forging of a HAp and PLLA mixture at elevated temperature can yield biocomposite blocks of shapes and sizes suitable for fixing of bone defects [7]. Sintering of a HAp and PLLA mixture with sodium chloride can also yield HAp/PLLA blocks of controlled porosity [8]. Hot and cold pressing of highly porous HAp/PLLA yields blocks of material of desired porosity and mechanical characteristics. The blocs with mechanical properties similar to bone tissue can be obtained by hot pressing (compressive strength $140 \mathrm{MPa}$ and module $10 \mathrm{GPa}$ ) [9-11]. Composite biomaterial HAp/PLLA, shaped as blocks, can also be obtained by injection molding procedure [12].

In vitro studies using HAp/PLLA composite biomaterial, although not reflecting the real-life process, taking place in an organism after implantation, can be a solid base for further material biocompatibility investigations. Changes, occurring under in vitro conditions, are in the first place associated with the PLLA degradation and its effects on PLLA molecular mass and mechanical characteristics [7]. Real behavior of the HAp/PLLA composite biomaterial can be discovered in vivo tests only. When implanted in an organism, HAp/PLLA biomaterial is exposed to the activity of complex enzyme systems and metabolism. Definition of this process gives a real picture of the material behavior and its characteristics [13].

IR as well as Fourier transform infrared (FT-IR) spectroscopy has been widely used in chemistry, physics and engineering [14]. Synthesis of composite biomaterials based on HAp was to date also analyzed in detail using FT-IR spectroscopy [15]. Medicine, with its specific investigations, offers really wide field for FT-IR spectroscopy application $[16,17]$. This technique was also successfully used for the obtaining of the important protein structural $[18,19]$. Tissue and biofluid diagnostics in vivo using FT-IR spectroscopy promotes new research fields in clinical diagnostic techniques. This method makes feasible characterization of the behavior of very complex protein and lipid molecules in real-life systems [20]. By this technique multicomponent solutions of biocompounds such as glucose, urea, and keratin with time dependent concentrations have been investigated as well [21]. FT-IR investigations in the field of medicine also carry within number of particularities that need attention during research [22].

In vivo research performed on the rabbits was mostly related to the histopathological research of HAp/PLLA composite biomaterials. In this research the mechanical hardness of the HAp/PLLA blocks was monitored during fifty weeks of implantation as well as the changes in crystallinity of PLLA [2326]. Out of HAp/PLLA composite biomaterial, mini plates were made that were analyzed in vivo. In this research the changes in mechanical properties and molecular weight were followed during the period of the implantation [27]. This kind of composite was also tested on rats [28,29]. HAp/PLLA biomaterial used in vivo can also be analyzed by FT-IR spectroscopy. Behavior of HAp/PLLA with PLLA of $50,000 \mathrm{~g} / \mathrm{mole}$ molecular weight and formation of connective collagen tissue which substitutes bioresorptive PLLA have been determined [30].

Until now, spectroscopy research did not examine the application of HAp/PLLA biomaterials during in vivo conditions. In this paper the bone tissue repair process in vivo using HAp/PLLA composite biomaterial was studied by FT-IR spectroscopy, scanning electron microscopy (SEM) and histopathologic analysis. Behavior of HAp/PLLA biomaterial of two compositions: one with PLLA of relatively low $(50,000 \mathrm{~g} / \mathrm{mole})$ and the other of high $(430,000 \mathrm{~g} / \mathrm{mole})$ molecular weight was analyzed. During the process, formation of new compounds and functional groups within them were detected by FT-IR 
spectroscopy. Possible induction of a new connective tissue - collagen using HAp/PLLA composite biomaterial with PLLA of relatively low and high molecular weights, especially important from the point of view of the biomaterial activity mechanism, was investigated. SEM and histopathologic analysis were used as the support for the received FT-IR results in the final phases of the research. Moreover, we examined the new application of FT-IR spectroscopy during in vivo test.

\section{Material and methods}

The components of HAp/PLLA composite biomaterial, HAp and PLLA of molecular weights of 50,000 and 430,000 g/mole, were synthesized in our laboratory. High-crystal phase of HAp was synthesized, while PLLA of both molecular weights was synthesized by polymerization of L-lactide using a non-toxic initiator. Cold pressing of the HAp and PLLA mixture gave biocomposite blocks as described earlier in detail $[4,5]$. For implantation, HAp/PLLA biocomposite of two different compositions, one with PLLA of 50,000 g/mole and the other with PLLA of 430,000 g/mole molecular weight further denoted as HAp/PLLA(50) and HAp/PLLA(430), respectively, was used. Cylindrical blocks of both compositions of a height of $1.5 \mathrm{~mm}$ and diameter of $1 \mathrm{~mm}$ were implanted.

$\mathrm{Balb} / \mathrm{c}$ Singen mice were used for in vivo experiments. HAp/PLLA(50) and HAp/PLLA(430) composite biomaterial samples were implanted intraperitoneally. In all cases, the HAp/PLLA consisted of 80 mass\% HAp and 20 mass\% PLLA. Experiments were performed using 40 young Balb/c male mice divided into two experimental groups. HAp/PLLA(50) biocomposite blocks were implanted into the first and HAp/PLLA(430) into the second group of mice. After one, three and twelve weeks, the implants were extracted and outer connective tissue layers removed.

This type of application, intraperitoneal, was chosen for its simplicity, frequent use in biocompatibility studies, and because peritoneal cavity could be a milieu for ectopic osteogenesis [26].

\subsection{FT-IR analysis}

After vacuum drying, the samples were ground and mixed with $\mathrm{KBr}$. The samples were then pressed into pellets suitable for FT-IR spectroscopy analysis using a Perkin Elmer 782 spectrometer. The spectral range from 400 to $4000 \mathrm{~cm}^{-1}$ was analyzed.

\subsection{SEM analysis}

Microstructure of HAp/PLLA biocomposite surface before and after implantation was observed by scanning electron microscopy (SEM) using a JSM 5300 JEOL. The samples were fixed in $0.4 \%$ glutaraldehyde for 24 hours, and then washed by a 0.2 M PBS (phosphate buffer solution) ( $\mathrm{pH}$ from 7.2 to 7.4). Postfixing was performed with $1 \%$ osmium tetroxide in a Veronal buffer ( $\mathrm{pH}$ from 7.2 to 7.4) for 2 hours. Fixed samples were dehydrated in graded concentrations of alcohol and then of acetone. After dehydration, the samples were dried in liquid $\mathrm{CO}_{2}$ at a critical point. The dried samples were spattered by gold in an ionic evaporator JFC 1100E JEOL.

\subsection{Histopathologic analysis}

The samples of implants and surrounding peritoneum tissue were fixed in a Brasil-Bouin fixative for 3 hours and postfixed in a $4 \%$ formalin buffer over night. Fixed implants were decalcified electrolytically 
in an aqueous solution of $8 \mathrm{vol} \% \mathrm{HCl}$ and $10 \mathrm{vol} \%$ formic acid. Decalcification lasted for two hours at a $100 \mathrm{~V}$ voltage and $50 \mathrm{~mA}$ current. The tissue was then dehydrated in alcohol and fitted into paraplast. Sections 3-6 micrometers thick were cut and stained using HE and PAS method. For periodic-acid Schiff method, rehydrated paraffin sections were embedded in periodic acid for $30 \mathrm{~min}$, and then stained by a Shiff reagent for an additional $30 \mathrm{~min}$. Contrasting was made by hematoxylin for $8 \mathrm{~min}$. Dehydrated sections were mounted on a glass plate and then analyzed by a light microscope. Special attention was paid to the bonds between the implant and the surrounding tissue [29].

\section{Results and discussion}

The spectrum of an HAp/PLLA(50) biocomposite sample prior to implantation is presented in Fig. 1a. The spectrum is characterized by absorption bands arising from HAp and PLLA of 50,000 g/mole molecular weight, determined by analogy with FT-IR spectra of pure HAp and the same PLLA standard samples.

Absorption bands at $3572 \mathrm{~cm}^{-1}$ and $631 \mathrm{~cm}^{-1}$ are attributed to the $\mathrm{OH}^{-}$groups from HAp. Absorption bands with maxima at 1090, 1050, 602 and $572 \mathrm{~cm}^{-1}$ arise from the phosphate groups of HAp. Characteristic absorption bands at $1760 \mathrm{~cm}^{-1}$ and at about $2920 \mathrm{~cm}^{-1}$ are attributed to the stretching vibrations of the $\mathrm{C}=\mathrm{O}$ and $\mathrm{C}-\mathrm{H}$ group of PLLA, respectively. The spectrum of an HAp/PLLA(430) biocomposite sample before implantation is shown in Fig. 1b. Almost the same absorption bands as for HAp and PLLA shown in Fig. 1a can be seen.

The spectrum of an HAp/PLLA(50) sample registered one week after in vivo test is presented in Fig. 2a. The spectrum contains already mentioned absorption bands arising from HAp/PLLA(50) biocomposite (as illustrated in Fig. 1a) and some new ones - a wide absorption band in the region 3000$3630 \mathrm{~cm}^{-1}$ and sharp absorption bands in the region $1470-1870 \mathrm{~cm}^{-1}$.

The bone healing process consists of an inflammation, repair and remodeling stage. We focused on the repair stage during which changes crucial for the synthesis and application of HAp/PLLA biocomposite took place. Beginning of the collagen phase formation is characterized by an increase in concentration of glucosamineglucan, a secondary amine comprising $\mathrm{NH}$ group in its structure [31]. Synthesis of collagen proceeds via enzyme and begins with the formation of short polypeptide chains. Two groups of polypeptides, pro- $\alpha 1$ and pro- $\alpha 2$, are synthesized by enzymes, mostly from $\alpha$-amino acids such as serine, proline and hydroxyproline [32]. The amino acids are interlinked by peptide bonds (NH-CO). Therefore, the following sequence of amino acids - glycin-proline-hydroxyproline - is predominantly present in collagen. Three polypeptide chains form procollagen which transforms into tropocollagen, a fundamental unit of collagen fibrils [32]. Collagen synthesis can be quantified by the appearance of proline, its labeling and recognition in the collagen synthesis steps [33].

According to the given FT-IR spectrum analysis of the protein structures, it is necessary to observe first of all the absorption bands arising from the $\nu(\mathrm{C}=\mathrm{O})$ stretching vibrations, so-called amide I bands, which appear at about $1650 \mathrm{~cm}^{-1}[16,19]$. Generally, amide I bands originate from the $\nu(\mathrm{C}=\mathrm{O})$ stretching vibrations coupled to $\delta(\mathrm{N}-\mathrm{H})$ bending vibrations. The amide II bands usually arise from the $\delta(\mathrm{N}-\mathrm{H})$ bending vibrations coupled to $\nu(\mathrm{C}-\mathrm{N})$ stretching vibrations. Namely, it is known that the amide I and II bands are present in the spectral $1500-1700 \mathrm{~cm}^{-1}$ range for protein molecules, first of all collagen $[16,19]$. In secondary amines, one absorption band is seen, arising from the stretching vibrations of $\mathrm{NH}$ group, of a medium to weak intensity, at $3350-3300 \mathrm{~cm}^{-1}$ or $3450 \mathrm{~cm}^{-1}$ [33]. The amide I band at 


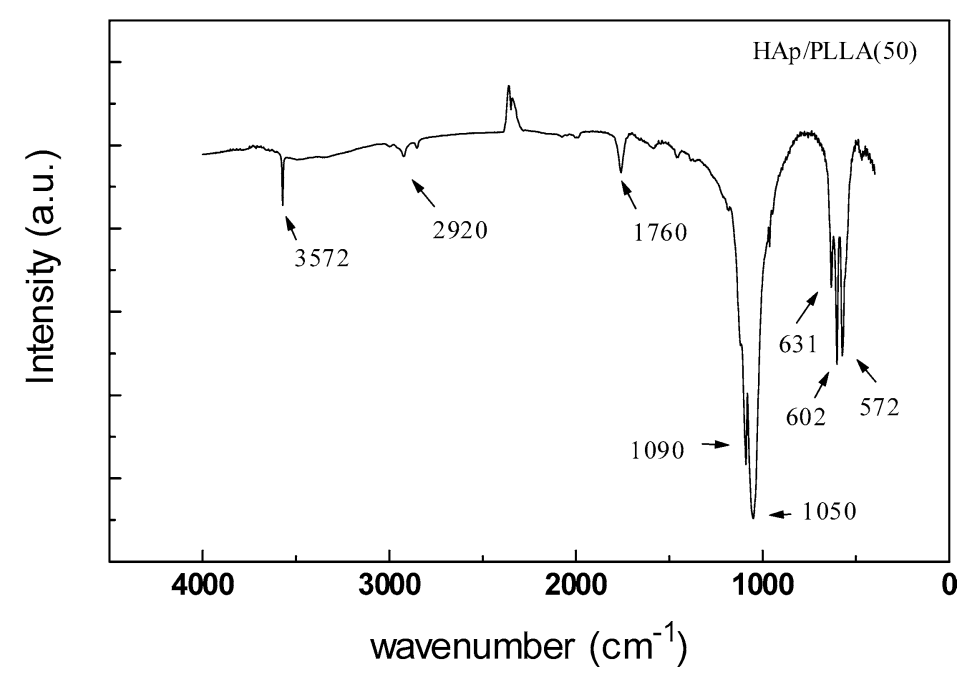

(a)

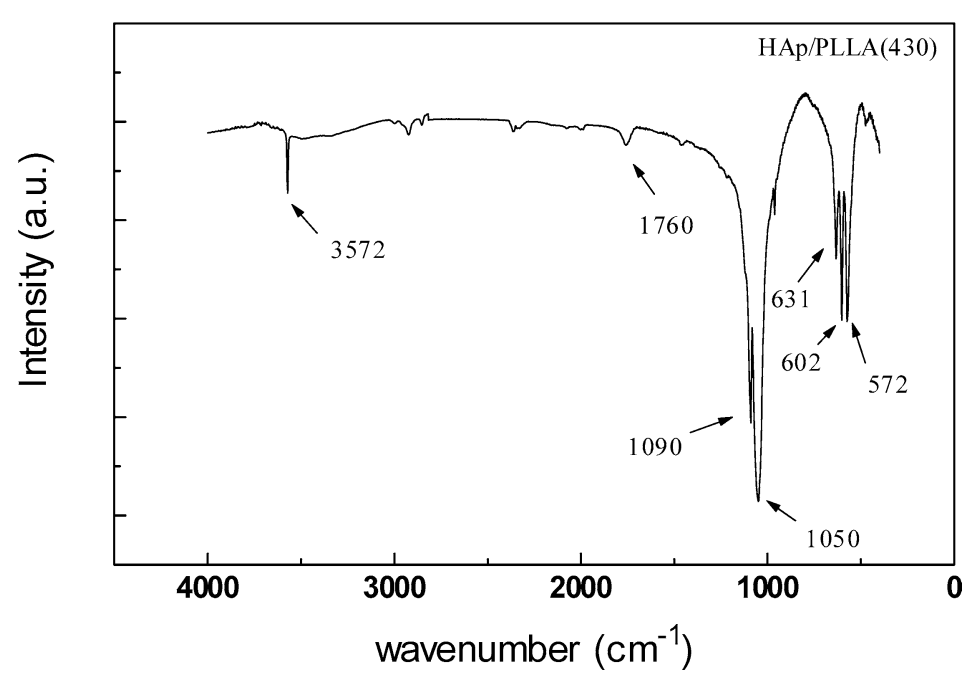

(b)

Fig. 1. IR spectrum sample before implantation: (a) HAp/PLLA(50), (b) HAp/PLLA(430).

about $1650 \mathrm{~cm}^{-1}$ can be seen in Fig. 2a. No absorption bands in the $1800-2800 \mathrm{~cm}^{-1}$ range are registered, characteristic of biological materials [20]. The repair stage begins with the appearance of secondary amines, as given earlier. Absorption bands at about $3310 \mathrm{~cm}^{-1}$ originate most probably from the stretching vibration of the $\mathrm{NH}$ group of the secondary amines. $\alpha$-amino acids gave characteristic spectra defined by the following absorption bands: a wide band of medium intensity at $3100-2600 \mathrm{~cm}^{-1}$ from the $\mathrm{NH}_{3}^{+}$stretching vibration, so-called "ammoniacal band"; and two weak bands at $1660-1590 \mathrm{~cm}^{-1}$ and $1550-1480 \mathrm{~cm}^{-1}$ arising from the asymmetric and symmetric $\mathrm{NH}_{3}^{+}$bending vibrations, respectively [34]. Absorption bands in the spectral region $1470-1870 \mathrm{~cm}^{-1}$, Fig. 2a, arise most probably from the new-formed $\alpha$-amino acids taking part in collagen synthesis. Appearance of the absorption bands between 1250 and $1500 \mathrm{~cm}^{-1}$ (Fig. 2a) may be attributed to the probably formed $\mathrm{CH}_{2}$ and $\mathrm{CH}_{3}$ groups 


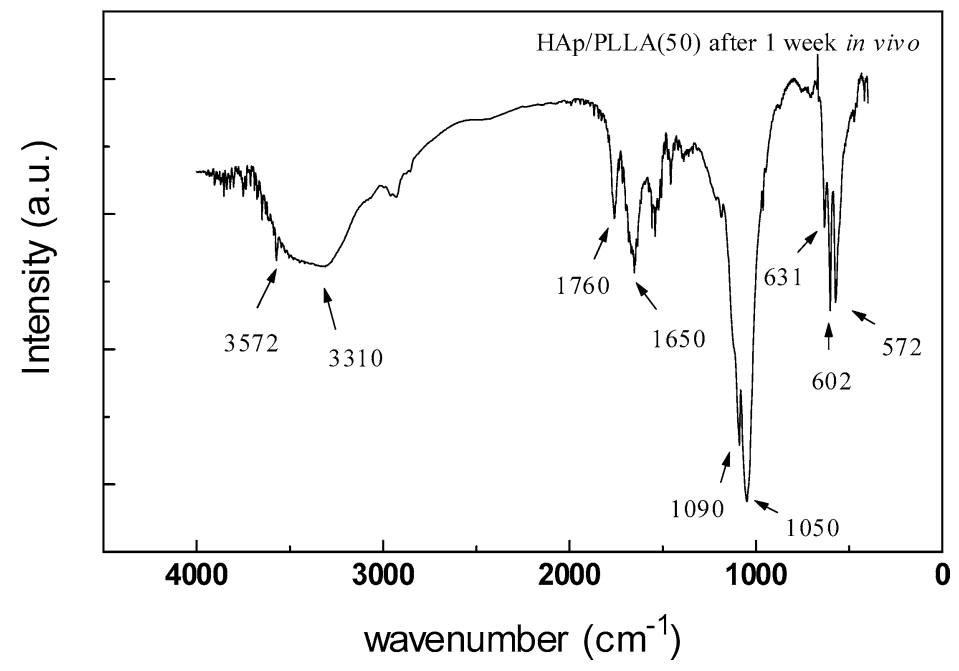

(a)

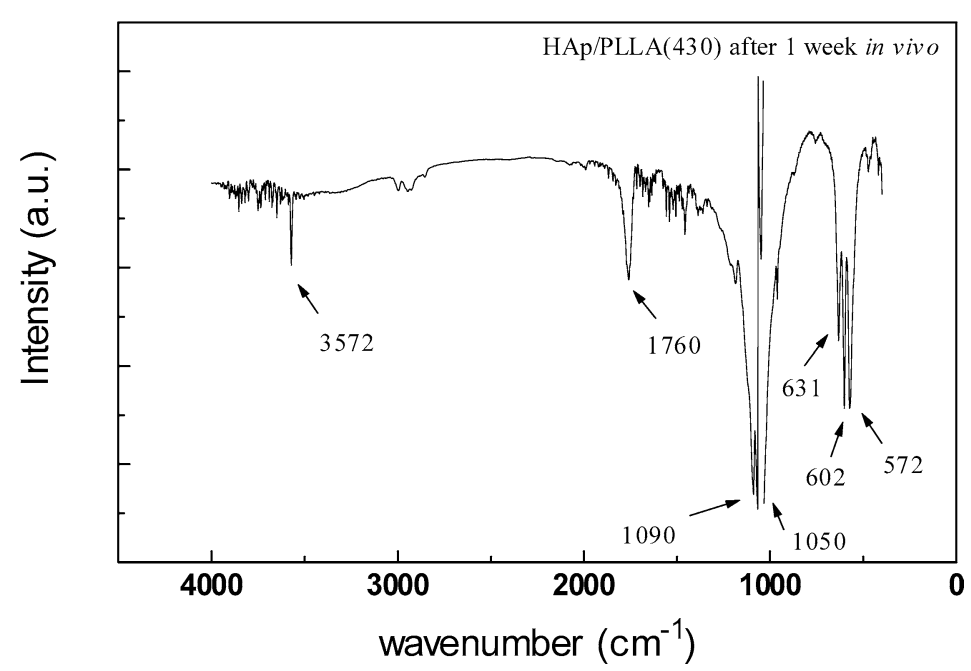

(b)

Fig. 2. IR spectrum sample after 1 week of testing in vivo: (a) HAp/PLLA(50), (b) HAp/PLLA(430).

of the lipid base, which are characteristic of biological materials [20]. Figure $2 b$ illustrates a spectrum of HAp/PLLA(430) material obtained one week after its implantation. Analysis of the spectrum did not reveal any significant difference from the spectrum of the same material given in Fig. 1b. Much greater changes are observed in the spectrum of HAp/PLLA(50) sample shown in Fig. 2a. Weak absorption bands, however, can be seen in the spectral range $1500-1700 \mathrm{~cm}^{-1}$ arising most probably from $\alpha$-amino acids formed one week upon implantation. Comparing the spectra presented in Figs $2 \mathrm{a}$ and $2 \mathrm{~b}$ it becomes clear that the biological processes proceed much faster with the HAp/PLLA(50) system than with HAp/PLLA(430) during the first week of in vivo tests.

Figure 3 shows the surface of the HAp/PLLA(50) composite biomaterial one week after the implantation. Very good adhesion of the connecting tissue cells can point out good biocompatibility of this 


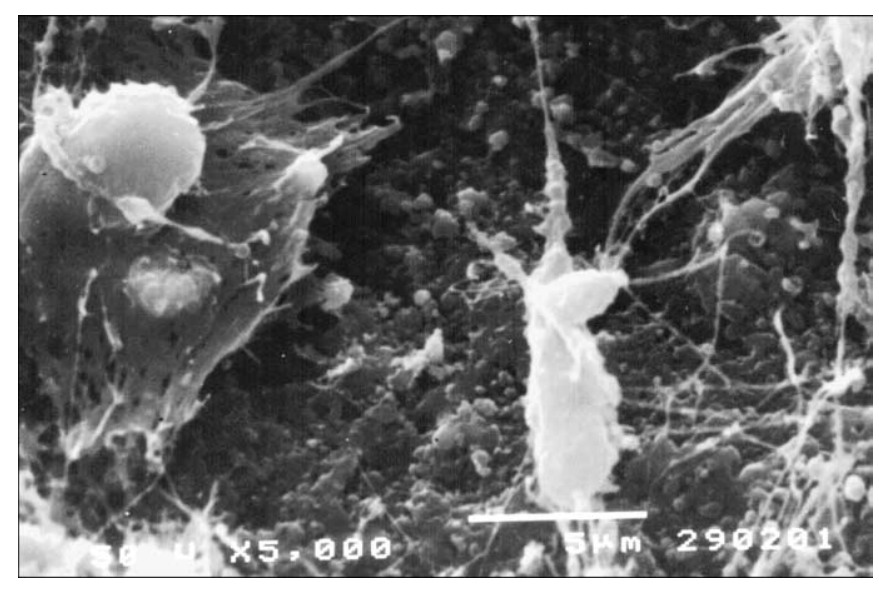

Fig. 3. The surface of HAp/PLLA(50) composite biomaterial after one week in vivo.

material. Good adhesion of the reparatory cells is the first step that enables the transduction and transcription in order to proliferate and differentiate the reparatory cells. Most probably macrophage (peritoneal macrophage) is discernable on the left side of the figure and fibroblast on the right side. Macrophage that is well adhered to the HAp/PLLA(50) surface, points out the possible phagocytosis that could be done. Fibroblast as the connecting tissue cell is also very well adhered to the surface. Possible good adhesion of fibroblast suggest his biocompatibility with the surface of this material, as well as the possible production of collagen by these cells. Potential collagen production by these cells suggests that the connecting tissue cells can successfully inhabit the surface of this material.

The spectrum obtained from an HAp/PLLA(50) sample three weeks upon implantation is presented in Fig. 4a. The spectrum is characterized by the presence of previously mentioned absorption bands from HAp/PLLA(50) and a wide and dominant absorption band at $3420 \mathrm{~cm}^{-1}$, which corresponds to the IR spectrum of collagen having a wide absorption band with a maximum at $3420 \mathrm{~cm}^{-1}$ [35]. The absorption band at $3420 \mathrm{~cm}^{-1}$ can be ascribed to the presence of $\nu(\mathrm{N}-\mathrm{H})$ vibrations of collagen and the band's width to the existence of $\nu(\mathrm{OH})$ vibrations also of collagen [20,34,35]. Accordingly, we can conclude that a connective tissue was generated 3 weeks upon implantation of HAp/PLLA and that the repair stage approaches its final step. Absence of the absorption bands in the region $1800-2800 \mathrm{~cm}^{-1} \mathrm{can}$ also be noticed confirming biological nature of the sample [14]. The spectrum of an HAp/PLLA(430) sample three weeks upon implantation is shown in Fig. 4b.

Absorption band of low intensity at about $1760 \mathrm{~cm}^{-1}$, slightly indicates its incomplete bioresorption. The absorption band at about $1650 \mathrm{~cm}^{-1}$ is present but weak compared to the other bands. Also, a wide absorption band without clearly seen maximum at about $3420 \mathrm{~cm}^{-1}$ is evident. According to the results obtained during the period of three weeks using HAp/PLLA(430) as an implant material, collagen was not generated but the compounds proceeding its generation. The stage reached using HAp/PLLA(430) (illustrated in Fig. 4b) three weeks upon implantation is very similar to that reached using HAp/PLLA(50) but one week upon implantation (shown in Fig. 2a).

Figure 5 shows the composite biomaterial spectrum twelve weeks after the implantation. The appearance of the numerous new absorption bands is evident. The composite biomaterial HAp/PLLA(50) spectrum, beside the earlier mentioned absorption bands, has a number of new ones, as shown on the Fig. 5a. Most probably the generation of the number of new protein and lipid structures transpired, which suggest the complexity of the obtained spectrum interpretation. Figure 5b shows the HAp/PLLA(430) 


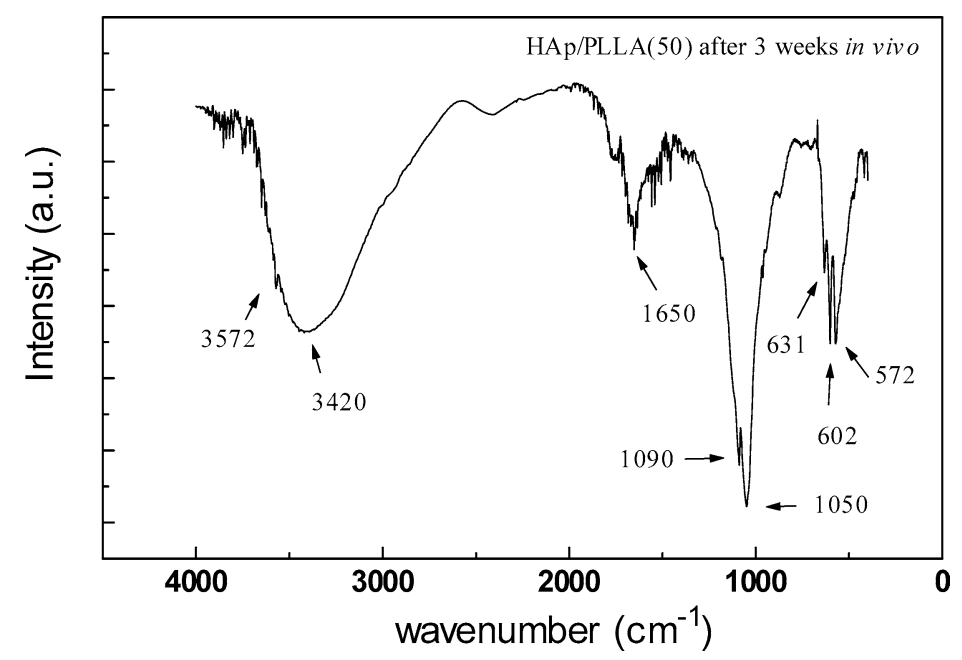

(a)

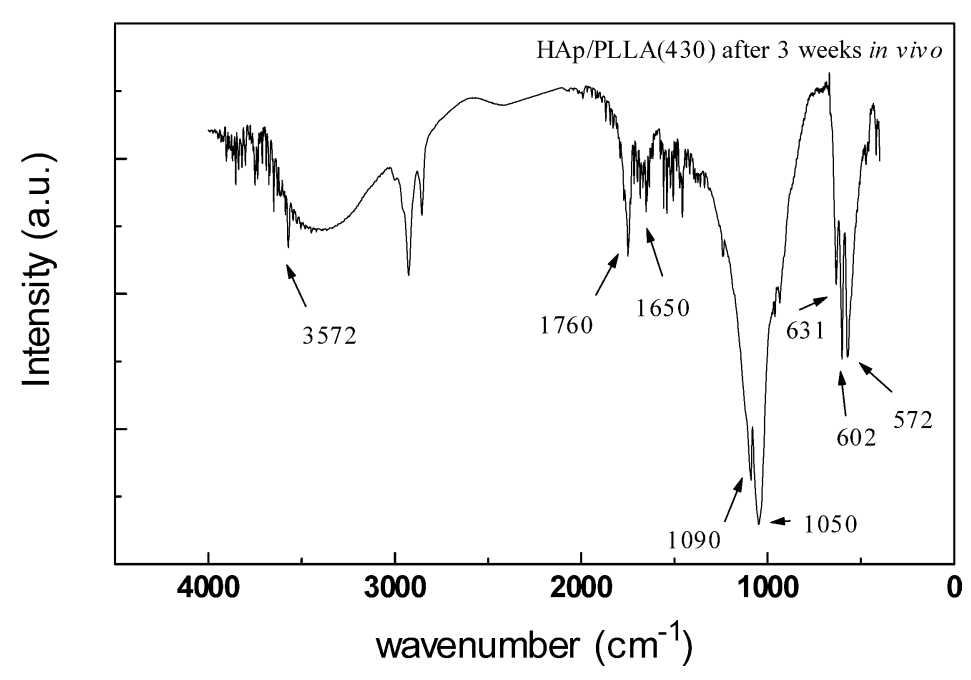

(b)

Fig. 4. IR spectrum sample after 3 weeks of testing in vivo: (a) HAp/PLLA(50), (b) HAp/PLLA(430).

composite biomaterial spectrum. The PLLA absorptive bands are noticeable on the Fig. 5b, which suggests that its bioresorption did not occur. The appearance of the collagen is also not noticeable. The appearance of the row of new absorptive bands, especially in the region from $1100-1800 \mathrm{~cm}^{-1}$, points out the generation possibility of the number of new compounds with protein and lipid structure.

Use of HAp/PLLA(50) blocks in vivo induced collagen generation as found after 3 weeks. Only based on a large number of samples, some statistical conclusion can be drawn. Where and how collagen generation took place within the biocomposite blocks could not be, however, determined by FT-IR spectroscopy. Because of this, in the early research the microstructure of HAp/PLLA(50) composite biomaterial was analyzed by SEM spectroscopy [30]. Based on earlier obtained results, it was established that after three weeks of in vivo research, between the HAp granules, instead of PLLA that was mostly re- 


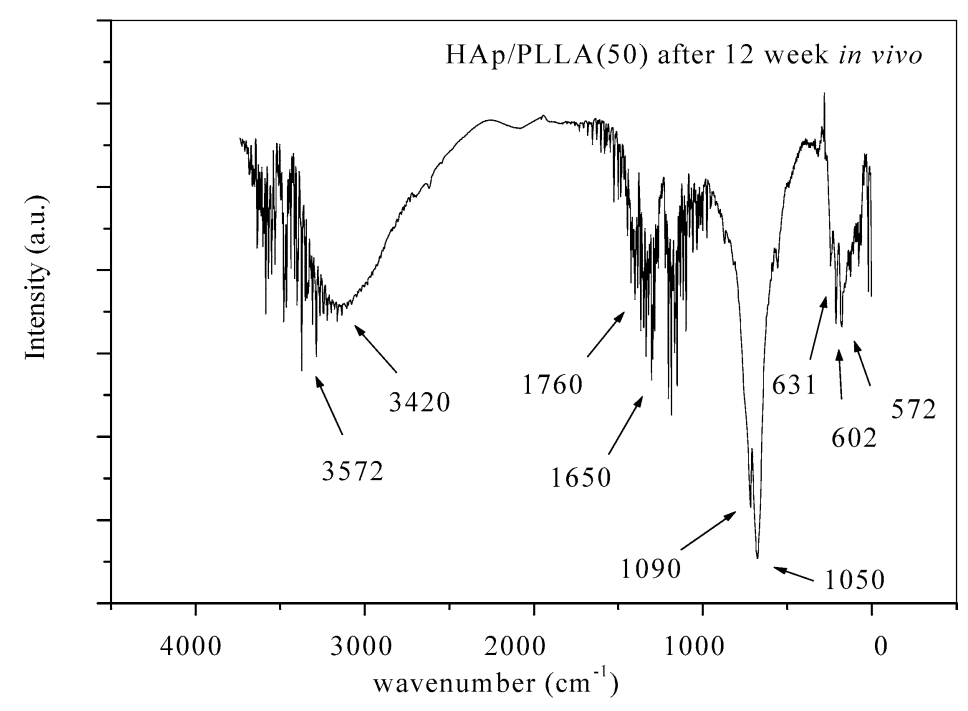

(a)

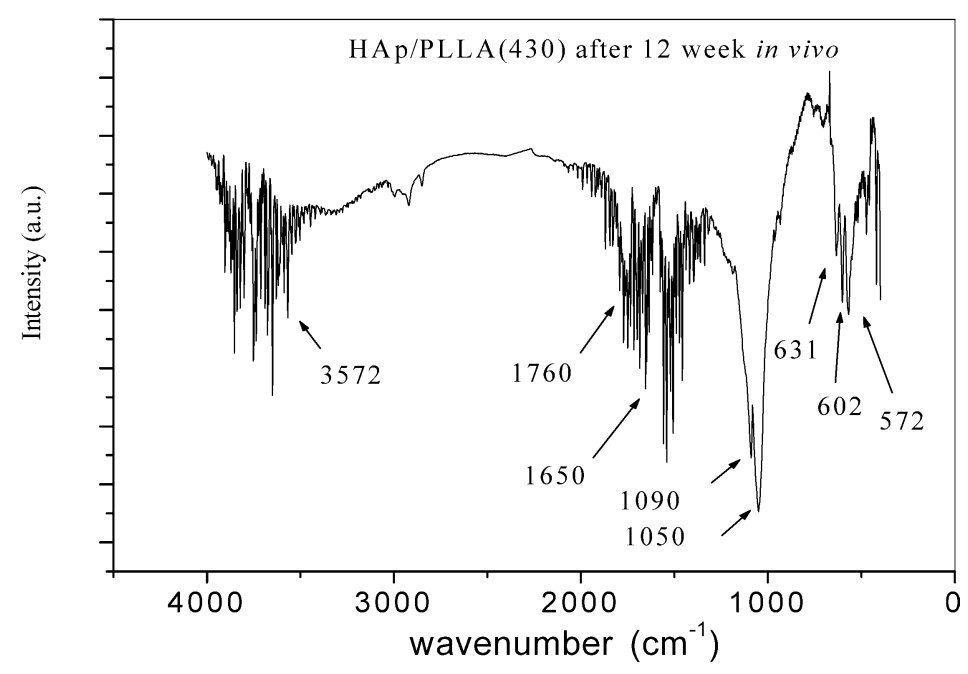

(b)

Fig. 5. IR spectrum sample after 12 weeks of testing in vivo: (a) HAp/PLLA(50), (b) HAp/PLLA(430).

sorbed, there were the connecting tissue fibers, most probably collagen. In case of HAp/PLLA(430) the connecting tissue fibers were not present. Obtained SEM results confirmed the earlier analyzed FT-IR research.

The longest time period of the HAp/PLLA composite biomaterial testing in this research is 12 weeks. Therefore, the samples analyzed by FT-IR spectroscopy after the longest time period, were histopathologicly analyzed also. Figure 6 shows the images obtained by histopathologic analysis after the implantation.

The particles of HAp/PLLA(50) composite biomaterial are homogeneous and pale eosinophilic. Wide gaps between the particles and the connective tissue are evident. Connective tissue between the particles 


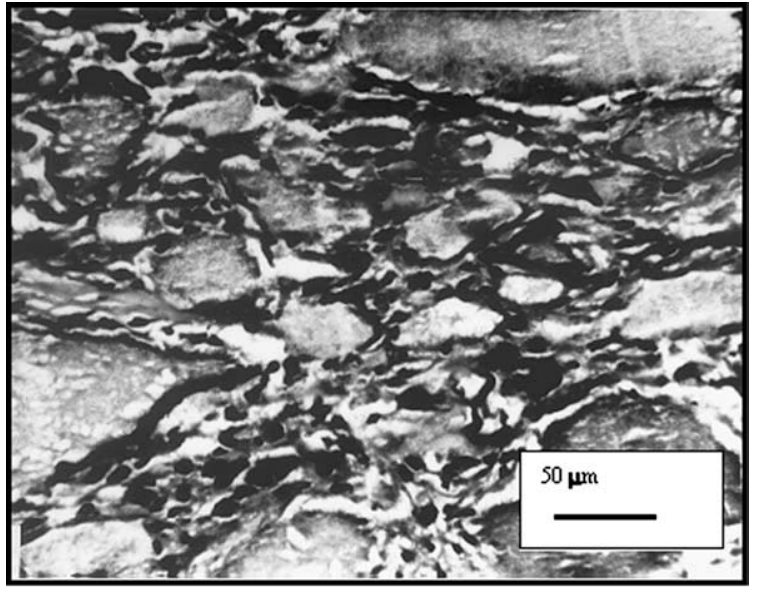

(a)

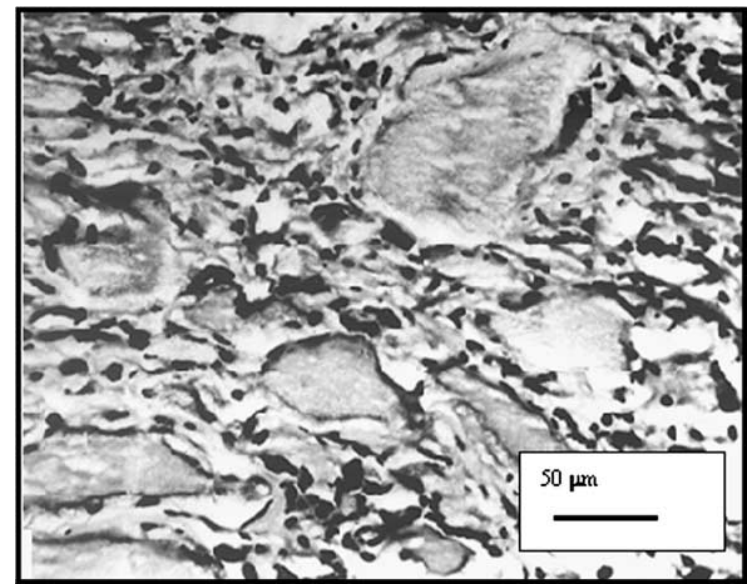

(b)

Fig. 6. Histopathological image twelve weeks after implantation: (a) composite biomaterials HAp/PLLA(50), (b) composite biomaterials HAp/PLLA(430).

consists mostly of small mononuclear cells, somewhat larger ones located next to the very implant. There are also composite particles whose central parts are well resorbed, while the periphery preserved the implant structure characterized by small grained pale eosinophilic material. A lot of cells with large hyperchromic nucleus can be seen immediately next to the implant surface, while the connective tissue in its depth contains cells of fibrocyte type and small hyperchromic ones with spherical nucleus and poor cytoplasm. The dominant connective tissue consists of fybroblasts mainly, and this corresponds to the fibrosis (Fig. 6a).

Narrow and wide bands of connective tissue are around implanted particles of HAp/PLLA(430) composite biomaterial separating them (Fig. 6b). Connective tissue near to the composite contains large multinuclear cells and beneath these cells there are gaps resulting probably from composite resorption. Small groups of large mononuclear and multinuclear cells are evident on several places, which cytoplasm as fingers get into the composite depth. The resorption on the composite periphery is the most intense, while the inner part still preserves small-grained pale-eosinophilic structure of the composite.

There are several reports on HAp as an inflammatory agent [36]. Also, a lot of data proved PLLA to be a cause of inflammatory response [37]. Inflammation was expected in our case too, due to insertion of a foreign body into peritoneum. Multinuclear cells observed on the preparations confirmed the presence of inflammatory process. Intense phagocytosis, observed even 12 weeks after implantation, on implanted particulates of HAp/PLLA(430), but weak in the case of HAp/PLLA(50), results probably from the difference in PLLA polymer structure. Long lasting phagocytosis may be a characteristic of long PLLA degradation [33]. The presence of PMN leukocytes on analyzed preparations is an intraperitoneal response to PLLA insertion. Inflammatory response, as stated earlier [38], is characterized by the largest increase in neutrophils 48 hours upon intraperitoneal injection of different PLLA particulates. Besides chemical composition of implants other data should also be taken into account. For instance some characteristics of inflammatory response do not show when other models of PLLA implantation are applied $[39,40]$.

As the studied synthetic materials behave as the natural bone, i.e., they are phagocytosed and resorbable, they can be considered as biocompatible. Resorption is usually the first step in a series of 
successive processes of bone remodeling and the osteogenesis is their physiological finale. HAp/PLLA composite biomaterials of different types and resorption rates could be designed and programmed to be suitable for particular purposes in an organism.

Different crystallinity of PLLA of various molecular weights may be the main reason for obtained different bioresorption of HAp/PLLA biocomposite materials. Sarasua et al. have shown that PLLA of higher molecular weight form bigger sferulites, with higher melting points and higher degree of crystallinity [41]. Furukawa et al. [23,24] have experimentally confirmed that HAp/PLLA composites with PLLA of lower molecular weight, after the implantation have higher degree of indicated affinity and faster degradation from PLLA of higher molecular weight.

\section{Conclusion}

Characteristic absorption bands, registered and defined by FT-IR spectroscopy, confirm the formation of new functional groups and compounds during the bone repair process using HAp/PLLA biocomposite with PLLA of 50,000 and 430,000 molecular weights. By this method, basic phenomena occurring in HAp/PLLA biocomposite, with PLLA of different molecular weights, tested in vivo can be determined. Appearance of the absorption bands at about 1650 and $3420 \mathrm{~cm}^{-1}$ arising from the material after implantation indicates generation of the connective tissue of collagen, not present at the beginning of the experiments. This generation is much faster when HAp/PLLA with PLLA of 50,000 g/mole molecular weight was used. Bioresorption and disappearance of PLLA took place upon implantation of HAp/PLLA biocomposite with PLLA of $50,000 \mathrm{~g} / \mathrm{mole}$ molecular weight. The process of PLLA bioresorption is characterized by a decrease and disappearance of the absorption bands at $1760 \mathrm{~cm}^{-1}$ arising from the $\mathrm{C}=\mathrm{O}$ group of PLLA. Bioresorption of PLLA, during the test period, when HAp/PLLA with PLLA of $430,000 \mathrm{~g} / \mathrm{mole}$ molecular weight was used is considerably slow.

Adhesion on the surface of the composite biomaterial, most probably osteoblast, suggests good biocompatibility.

Histopathologic research suggested that the studied implants do not cause a pronounced inflammatory response. Their good ingrowth into the surrounding connective tissue confirms their good biocompatibility. There is a difference in the behavior between the studied biocomposites regarding resorption rate and tissue response. The resorption of implants ranges in intensity from the highest with the bone implants, low in the case of HAp/PLLA(50), to the lowest with the HAp/PLLA(430) implants. After 12 weeks, the resorption of HAp/PLLA(50) is minor, but still apparent with the HAp/PLLA(430) preparations. On the other hand, cellular response to HAp/PLLA(430) more resembles that to bone implants. When considering application of these materials, their bioactive properties would certainly have to be taken into account.

\section{Acknowledgements}

The authors are grateful to Prof. Dr. Steva Najman, Prof Dr Vojin Savic and Mr Ljubisa Djordjevic from the Faculty of Medicine, University of Nis, for their help in conducting in vivo tests. This work was supported by the Ministry of Science, Technology and Development of the Republic of Serbia, under Project No. 1431: Molecular designing of monolith and composite materials. 


\section{References}

[1] L. Hench, Bioceramics: from concept to clinic, J. Am. Cer. Soc. 74 (1991), 1487-1510.

[2] M. Bever, ed., Biodegradation of biomedical polymers, in: Encyclopedia of Materials Science and Engineering, Vol. 1, Pergamon Press, Oxford, 1986, pp. 323-326.

[3] J. Middeleton and A. Tipton, Synthetic biodegradable polymers as orthopedic devices, Biomaterials 21 (2000), 23352346.

[4] N. Ignjatovic, S. Tomic, M. Dakic, M. Miljkovic, M. Plavsic and D. Uskokovic, Synthesis and properties of hydroxyapatite/poly-L-lactide composite biomaterials, Biomaterials 20 (1999), 809-816.

[5] N. Ignjatovic, M. Plavsic and D. Uskokovic, Composite biomaterial hydroxyapatite/poly-L-lactide (collagen) with polyL-lactide of different molar weigth, Adv. Eng. Mater. 2 (2000), 511-514.

[6] C.C.P.M. Verheyen, C.P.A.T. Klein, J.M.A. de Blieckhogervorst, J.G.C. Wolke, C.A. van Blitterswijk and K. de Groot, Evaluation of hydroxyapatite/poly(l-lactide) composites: physico chemical properties, J. Mater. Sci.: Mat. Med. 4 (1993), $58-65$.

[7] Y. Shikinami and M. Okuno, Bioresorbable devices made of forged composites of hydroxyapatite (HA) particles and poly-l-lactide (PLLA): part I. Basic characteristics, Biomaterials 20 (1999), 859-877.

[8] C. Laurencin, M. Borden, M. Attawia, F. Ko and G. Morrill, Polymer based tissue engineering of bone, Polymer Preprints 39 (1998), 122-123.

[9] N. Ignjatovic, K. Delijic, M. Vukcevic and D. Uskokovic, The designing of properties of calcium-hydroxyapatite/polylactide composite biomaterials by hot pressing, Z. Metallkunde 92 (2001), 145-149.

[10] N. Ignjatovic, K. Delijic, M. Vukcevic and D. Uskokovic, Microstructure and mechanical properties of hot pressed hydroxyapatite/poly-l-lactide biomaterials, Key Eng. Mater. 192-195 (2001), 737-740.

[11] N. Ignjatovic, E. Suljovrujic, J. Budimski, I. Krakovsky and D. Uskokovic, Evaluation of hot pressed hydroxyapatite/polyL-lactide composite biomaterial, Journal of Biomedical Materials Research Part B: Applied Biomaterials 71B(2) (2004), article on line.

[12] L. Calandrelli, B. Immirizi, M. Malinconicio, M. Volpe, A. Oliva and F. Della Ragione, Preparation and characterization of composites based on biodegradable polymers for in vivo application, Polymer 41 (2000), 8027-8033.

[13] C. Cehreli, S. Sahin, K. Kesenci, K. Tuzlakogu, E. Piskin, S. Ozturuk, B. Caner and M. Bozkurt, Biological reaction to a poly(l-lactide)-hydroxyapatite composites: a study in canine mandible, Journal of Biomaterials Application 17 (2003) 265-276.

[14] B.P. Colarusso, L.H. Kidder, I.W. Levin, J.C. Fraser, J.F. Arens and E.N. Lewis, Infrared spectroscopic imaging: From planetary to cellular systems, Appl. Spectrosc. 52 (1998), 106-120.

[15] M. Chang and J. Tanaka, FT-IR study for hidroxyapatite/collagen nanocomposite cross-linked by glutaraldehyde, Biomaterials 23 (2002), 4811-4818.

[16] H.H. Mantsch and R.N. Mc Elhaney, Applications of infrared spectroscopy to biology and medicine, J. Mol. Struct. 217 (1990) 347-362.

[17] M. Huleihel, M. Talyshinsky and V. Erukhimovitch, FT-IR microscopy as a method for detection of retrovirally transformed cells, Spectroscopy 15 (2001), 57-64.

[18] P. Haris and D. Chapman, Does Fourier-transform infrared-spectroscopy provide useful information on protein structures?, Trends in Biochemical Sciences 17(9) (1992), 328-333.

[19] C. Chang and J. Tanaka, FT-IR study for hydroxyapatite/collagen nanocomposite cross-linking by glutaraldehyde, Biomaterials 23 (2002), 4811-4818.

[20] H. Mantsch and M. Jackson, Molecular spectroscopy in biodiagnostics, J. Mol. Struct. 347 (1995), 187-206.

[21] T. Fujii, Y. Miyahara and Y. Watanabe, Highly sensitive biochemical analysis using low-temperature infrared spectroscopy measurements, Appl. Spectrosc. 51 (1997), 1682-1686.

[22] V. Crupi, S. Galli, D. Majolino, P. Migliardo, P. Pergolizzi and V. Venuti, Recent results on biomedical problems: A Fourier transform infrared (FT-IR) study, Spectroscopy 16 (2002), 245-250.

[23] T. Furukawa, Y. Matsusue, T. Yasunaga, Y. Shikinami, M. Okuno and T. Nakamura, Biodegradation behavior of ultrahigh-strength hydroxyapatite/poly(l-lactide) composite rods for internal fixation of bone fractures, Biomaterials 21 (2000), 889-898.

[24] T. Furukawa, Y. Matsusue, T. Yasunaga, Y. Nakagawa, Y. Okada, Y. Shikinami, M. Okuno and T. Nakamura, Histomorphometric study on high-strength hydroxyapatite/poly(l-lactide) composite rods for internal fixation of bone fractures, J. Biomed. Mater. Res. $\mathbf{5 0}$ (2000), 410-419.

[25] T. Furukawa, Y. Matsusue, T. Yasunaga, Y. Nakagawa, Y. Shikinami, M. Okuno and T. Nakamura, Bone bonding ability of a new biodegradable composite for internal fixation of bone fractures, Clin. Orth. Rel. Res. 379 (2000), 247-258.

[26] S. Liao, In vitro and in vivo degradation of mineralised collagen based composite scaffold: nanohydroxyaptite/collagen/ poly(l-lactide), Tissue Engineering 10 (2004), 73-80. 
[27] Y. Shikinami and M. Okuno, Bioresorbable devices made of forged composites of hydroxyapatite (HA) particles and poly-l-lactide (PLLA). Part II: pratical properties of miniscrews and miniplates, Biomaterials 22 (2001), 3197-3211.

[28] W. Heidemann, S. Jeschkeit, K. Ruffieux, J. Fischer, M. Wagner, G. Kruger, E. Wintermantel and K. Gerlach, Degradation of poly(DL)lactide implants with or without addition of calciumphosphates in vivo, Biomaterials 22 (2001), 2371-2381.

[29] S. Najman, Lj. Dordjevic, V. Savic, N. Ignjatovic and D. Uskokovic, Biological evaluation of hydroxyapatite/poly-1lactide composite biomaterials with poly-l-lactide of different molecular weights intraperitonealy implanted into mice, Bio-Medical Materials and Engineering 14 (2004), 61-70.

[30] N. Ignjatovic, V. Savic, S. Najman, M. Plavsic and D. Uskokovic, A study of HAp/PLLA composite as a substitute for bone powder, using FT-IR spectroscopy, Biomaterials 22 (2001), 571-575.

[31] J. Linder, Bone healing, Clin. Plast. Surg. 4 (1977), 425-437.

[32] L. Stryer, Biochemistry, 2nd edn, W.H. Freeman and Company, San Francisco, 1981, pp. 186-204.

[33] W. El Harake, M. Furman, B. Cook, K. Nair, J. Kukowski and I. Brodsky, Measurement of dermal collagen synthesis rate in vivo humans, Am. J. Phys. End. Metab. 274 (1998), 586-591.

[34] J. Bellamy, The Infra Red Spectra of Complex Molecules, London, Chapman \& Hall, 1975, pp. 247-258.

[35] D.O. Hummel, Atlas of Polymer and Plastics Analysis, Second, completely revised edition, Carl Hansen Verlag, 1984, pp. 373.

[36] H. Schumacher, Crystals, inflammation, and osteoarthritis, Am. J. Med. 83 (1987), 11-16.

[37] R. Bos, F. Rozema, G. Boering, A. Nijenhuis, A. Pennings, A. Verwey, P. Nieuwenhuis and H. Jansen, Degradation of and tissue reaction to biodegradable poly(L-lactide) for use as internal fixation of fractures: a study in rats, Biomaterials 12 (1991), 32-36.

[38] A. van Sliedregt, M. Knook., S. Hesseling, H. Koerten, K. de Groot and C. van Blitterswijk, Cellular reaction on the intraperitoneal injection of four types of polylactide particulates, Biomaterials 13 (1992), 819-824.

[39] K. Hooper, T. Nickolas, E. Yurkow, J. Kohn and D. Laskin, Characterization of the inflammatory response to biomaterials using a rodent air pouch model, J. Biomed. Mater Res. 50 (2000), 365-374.

[40] J. Bergsma, F. Rozema, R. Bos, G. Boering, W. de Bruijn and A. Pennings, In vivo degradation and biocompatibility study of in vitro pre-degraded as-polymerized polylactide particles, Biomaterials 16 (1995), 267-274.

[41] J. Sarasua, R. Prud'homme, M. Wisniewski, A. Borgne and N. Spassky, Crystalization and melting behavior of polylactides, Macromolecules 31 (1998), 3895-3905. 


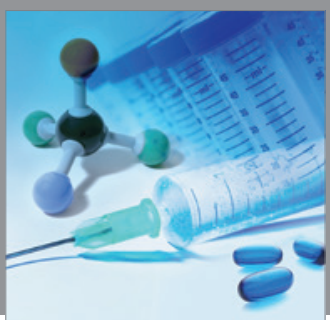

International Journal of

Medicinal Chemistry

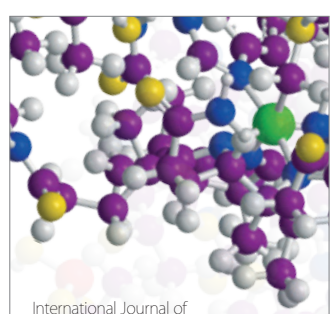

Carbohydrate Chemistry

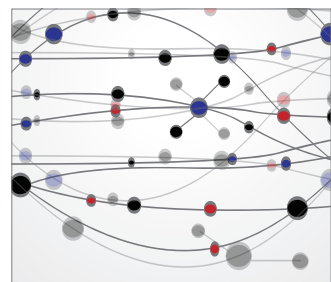

The Scientific World Journal
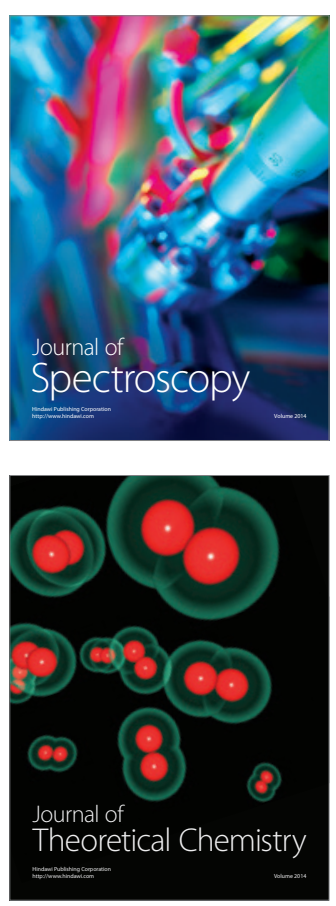
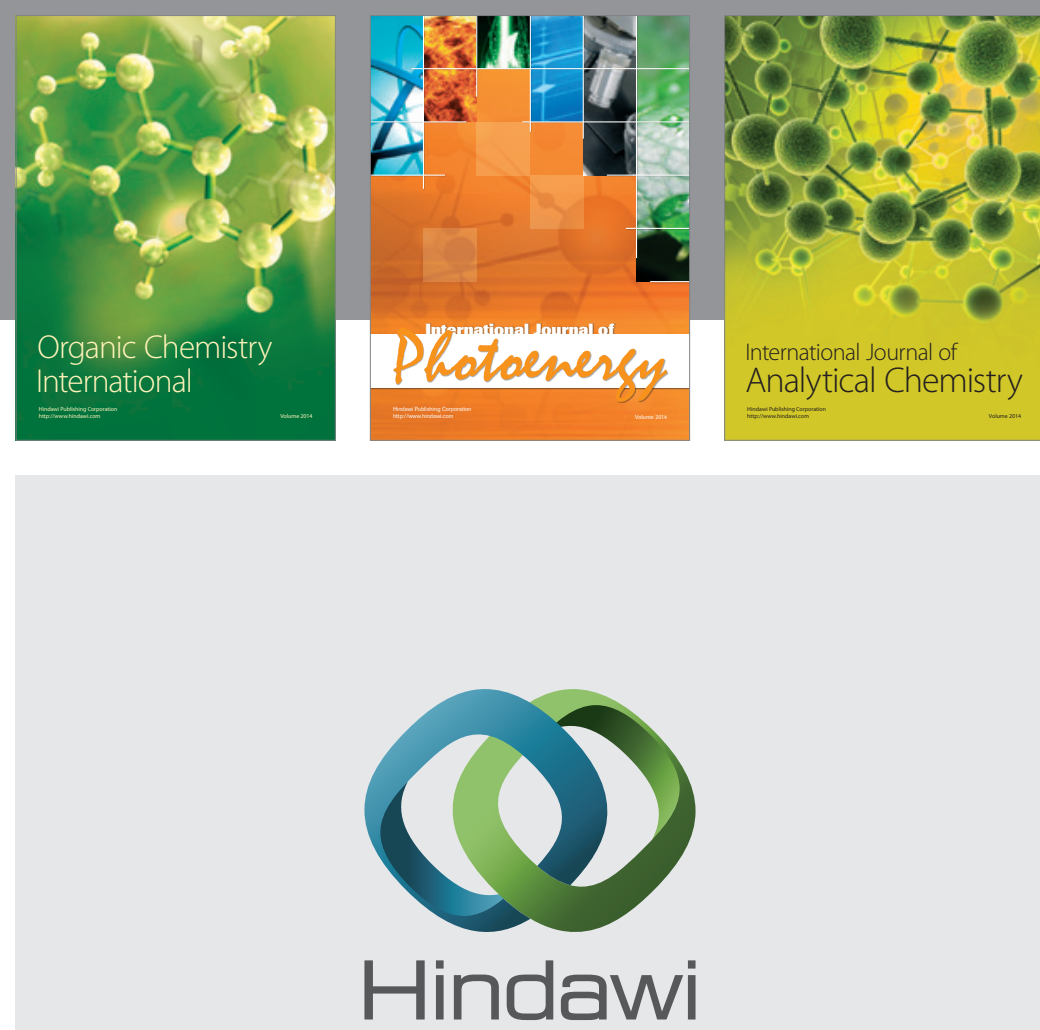

Submit your manuscripts at

http://www.hindawi.com
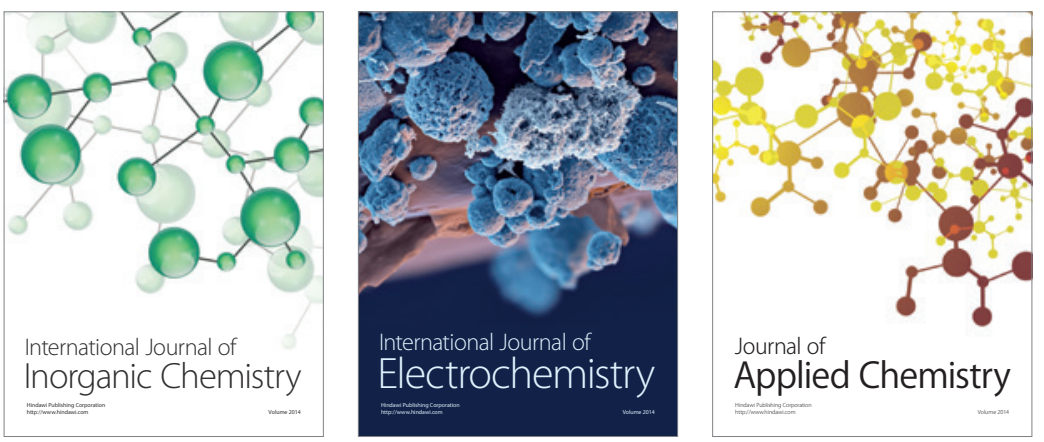

Journal of

Applied Chemistry
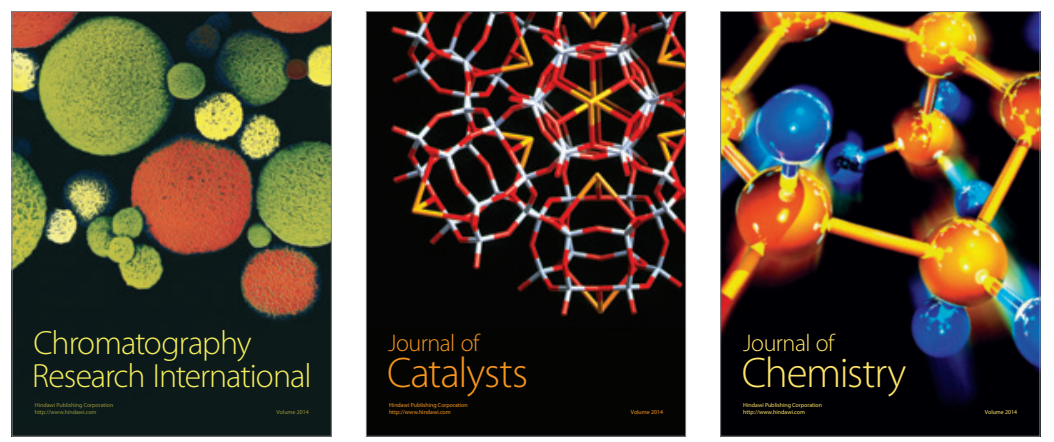
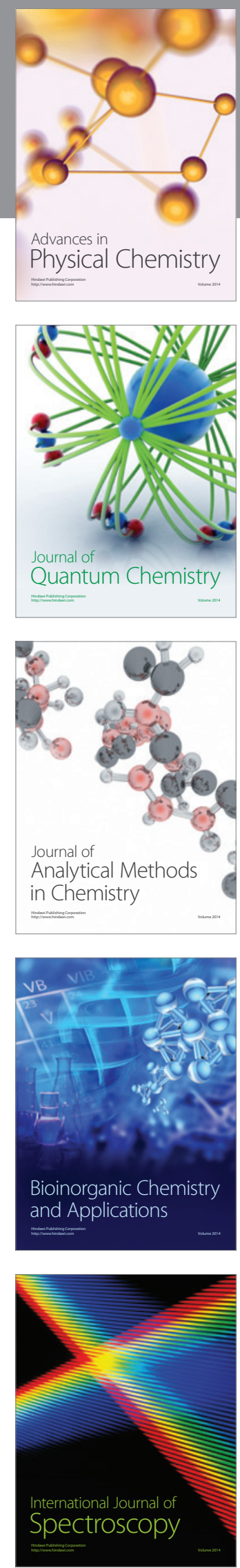